\title{
Twelve-month outcomes in patients with retinal vein occlusion treated with low-frequency intravitreal ranibizumab
}

This article was published in the following Dove Press journal:

Clinical Ophthalmology

21 June 2016

Number of times this article has been viewed

\section{Yoshihito Sakanishi \\ Ami Lee \\ Ayumi Usui-Ouchi \\ Rei Ito \\ Nobuyuki Ebihara}

Department of Ophthalmology, Juntendo University Urayasu Hospital, Urayasu City, Chiba, Japan
Correspondence: Yoshihito Sakanishi Department of Ophthalmology, Juntendo University Urayasu Hospital, 2-I-I Tomioka, Urayasu City, Chiba, 279-002I Japan

Tel +8I 473533 III

Email yossy1950@hotmail.com
Purpose: The purpose of this study was to determine the clinical efficacy of low-frequency intravitreal ranibizumab to treat macular edema due to retinal vein occlusion (RVO).

Patients and methods: This was a retrospective examination of cases that received intravitreal ranibizumab for untreated RVO over a period of 12 months. Instead of the conventional three monthly injections, injections were given once during the introductory period. If the recurrence of macular edema was diagnosed during the monthly visit, additional injections were given as needed. There were 21 eyes of 21 patients with branch RVO (BRVO) and ten eyes of ten patients with central RVO (CRVO). The parameters examined included the number of injections over the 12-month period, improvements in best-corrected visual acuity (BCVA), and the central macular thickness (CMT). For BRVO, preinjection parameters that had an effect on the prognosis of BCVA after the 12-month period were also examined.

Results: The total mean number of injections over the 12-month period was 3.4 for CRVO and 2.1 for BRVO. For CRVO, the BCVA in log minimum angular resolution changed from a preinjection value of 0.80 to 0.55 at 12 months. For BRVO, the change was from 0.51 to 0.30 . For all diseases, BCVA improved after 12 months compared with the preinjection values $(P<0.05)$. There was improvement in the CMT, and the CRVO changed from $765.0 \mu \mathrm{m}$ at preinjection to $253.5 \mu \mathrm{m} 12$ months later. BRVO changed from 524.1 to $250.1 \mu \mathrm{m}$, and preinjection BCVA was associated with a prognosis of visual acuity after 12 months of the initial injection $(P=0.0485)$.

Conclusion: Even with a low number of injections during the introductory period, there were still improvements in both visual acuity and CMT in RVO patients after 12 months, indicating that it was an effective treatment.

Keywords: retinal vein occlusion, IVR, macular edema, BRVO, CRVO

\section{Introduction}

Retinal vein occlusion (RVO) is the second most frequent type of retinal vascular disorder after diabetic retinopathy and is reported to affect an estimated 16 million people in the world. ${ }^{1-3}$ RVO vision loss is a result of macular edema (ME), whose primary cause is vascular endothelial growth factor (VEGF). Previously, ME caused by RVO has been reported in studies using steroid therapy and photocoagulation, ${ }^{5,6}$ but recently, effective therapies using anti-VEGF medications are being widely used..$^{7-11}$

We determined if the number of intravitreal injections could be minimized to decrease possible complications and the financial burden of the injections. Here, we report our results using intravitreal ranibizumab (IVR; Lucentis; Genentech, Inc., South San Francisco, CA, USA). We used one injection and pro re nata (PRN), instead of 
using the conventional three monthly injections during the same period.

\section{Patients and methods}

The patients for this retrospective study were consecutive cases of RVO who were diagnosed at the Juntendo University Urayasu Hospital, where they received IVR treatment and examinations over a period of 12 months after the initial injection. Cases where the patients received dosages of other anti-VEGF medications prior to the initial IVR were excluded from the study, as were cases with sub-Tenon's injections of triamcinolone, cases receiving retinal photocoagulation, or cases diagnosed with other macular diseases. Written informed consent was obtained from each patient who underwent treatment after an explanation of their their treatment.

A single injection of IVR $(0.5 \mathrm{mg} / 0.05 \mathrm{~mL})$ was administered during the introductory period. Afterward, the drug was readministered during a monthly visit if the central macular thickness (CMT) was $>300 \mu \mathrm{m}$ because of residual or reoccurring subretinal fluid. Best-corrected visual acuity (BCVA) was not used as a standard for readministration of IVR.

Fluorescein angiography was performed before initial injections. Cases where the capillary nonperfusion regions were greater than five optic disk areas in branch RVO (BRVO) and ten optic disk areas in central RVO (CRVO) were defined as ischemic. In cases with CRVO, as soon as ischemia was identified, panretinal photocoagulation (PRP) was performed in parallel with injections with the aim of preventing neovascular glaucoma.

The injection frequency of these cases over a 12-month period, including the times between the initial injections and reinjections, as well as the transitions in visual acuity and CMT, was examined 1 week before and after injections and at 1 month, 3 months, 6 months, and 12 months after the initial injection.

We also examined preinjection parameters that affected visual acuity after 12 months of the initial injection. The preinjection factors were as follows: sex, age, CMT on optical coherence tomography (OCT), BCVA, whether the patient was diagnosed with ischemia, and the presence or absence of an ellipsoid zone continuity on OCT. The BCVA was expressed as decimal visual acuity using a Landolt $C$ chart and converted to $\log$ minimum angular resolution (logMAR) for analysis. We employed Cirrus HD-OCT (Carl Zeiss Meditec AG, Jena, Germany) to measure CMT and judged the continuity of the ellipsoid zone using high-definition, five-line raster images.

This study was approved by the Ethics Committee of Juntendo University Urayasu Hospital. The study complied with the Health Insurance Portability and Accountability Act, and the procedures adhered to the tenets of the Declaration of Helsinki.

\section{Statistics}

Data were analyzed using StatView for Windows (SAS Institute Inc., Cary, NC, USA). We used the analysis of variance for comparisons at different time points before and after injections, as well as the Dunnett's test of multiple comparisons, and defined statistical differences as $P<0.05$.

We performed logistic regression analyses of preinjection parameters affecting BCVA at 12 months after the initial injections and considered a BCVA $<0.2$ on the $\log$ MAR scale after 12 months to be the dependent variable and preinjection parameters as the independent variables.

\section{Results}

For BRVO, 21 eyes of 21 patients aged 43-81 years (average age 65.1 years) were examined. For CRVO, ten eyes of ten patients aged 41-84 years (average age 66.1 years) were examined.

The injection frequency over the 12-month period was $3.4 \pm 1.8$ (mean \pm standard deviation) times for CRVO and $2.1 \pm 1.0$ times for BRVO.

When we compared the BCVA prior to initial injections with the BCVA after 12 months, we considered a change in $\operatorname{logMAR}$ of $>0.3$ as significant. For CRVO, four eyes (40\%) showed improvement, five $(50 \%)$ eyes showed no change, and one $(10 \%)$ eye worsened. For BRVO, ten $(47.6 \%)$ eyes improved, nine $(42.9 \%)$ eyes showed no change, and two $(9.5 \%)$ eyes worsened. No serious adverse ocular events were seen in any patient.

The period from initial injection to recurrence for CRVO was 1 month for three eyes, 2 months for one eye, 3 months for three eyes, 10 months for one eye, and no recurrence for two eyes. For BRVO, the period was 1 month for two eyes, 2 months for one eye, 3 months for six eyes, 4 months for two eyes, 5 months for one eye, 6 months for one eye, 8 months for one eye, 9 months for one eye, and no recurrence for six eyes.

For CRVO, the CMT improved from $765.0 \mu \mathrm{m}$ at preinjection to $253.5 \mu \mathrm{m} 12$ months later (Figure 1). For BRVO, the CMT improved from $524.1 \mu \mathrm{m}$ at preinjection to $250.1 \mu \mathrm{m} 12$ months later (Figure 2). We found significant improvements in all ocular disorders at all time points.

BCVA changed from a logMAR value of 0.80 at preinjection to 0.5512 months later for CRVO (Figure 3). Compared with preinjection, there was a significant improvement in BCVA at the 6- and 12-month time points. For BRVO, the 


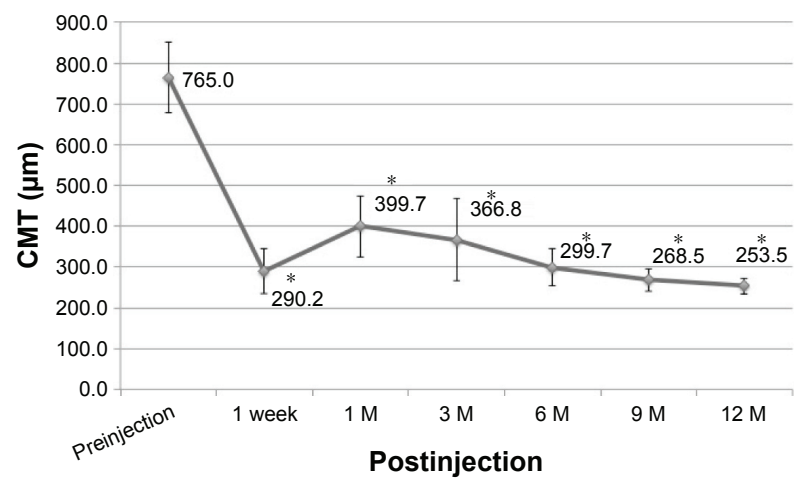

Figure I Changes in CMT in CRVO.

Note: ${ }^{*} P<0.05$, ANOVA versus preinjection.

Abbreviations: CMT, central macula thickness; CRVO, central retinal vein occlusion; ANOVA, analysis of variance; $M$, month.

change was from 0.51 at preinjection to 0.3012 months later (Figure 4). Compared with preinjection, there was a significant improvement in BCVA, with the exception of the 3-month time point.

We found that a good BCVA at preinjection was associated with a visual acuity of $<0.2$ on the $\log$ MAR scale after 12 months of the initial injection (Table 1).

\section{Discussion}

There have been different numbers of injections for intravitreal anti-VEGF during the introductory period for ME caused by RVO. Previous studies reported that anti-VEGF medications were injected three or six times during the introductory period. ${ }^{10-12} \mathrm{We}$ thought that the number of intravitreal injections should be minimized because of complications and because of possible financial burdens associated with the injections. We therefore reduced the treatment to one injection and $\mathrm{PRN}$, instead of the conventional three monthly loading injections. BCVA improvements after 12 months

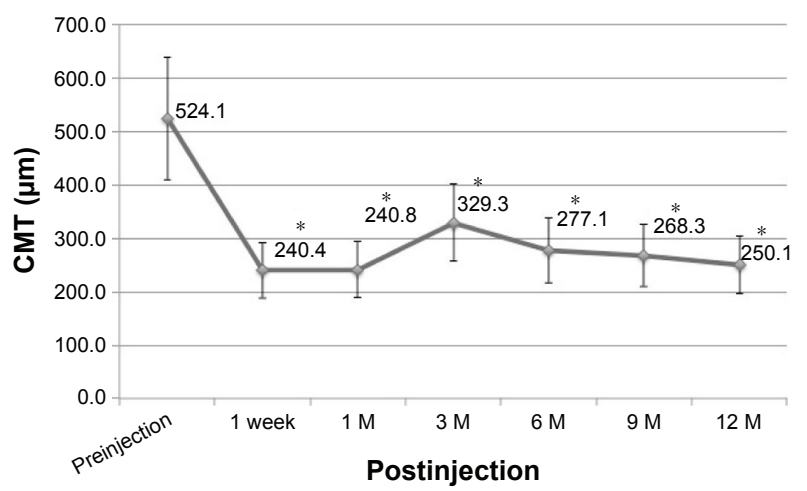

Figure 2 Changes in CMT in BRVO.

Note: $* P<0.05$, ANOVA versus preinjection.

Abbreviations: CMT, central macula thickness; BRVO, branch retinal vein occlusion; ANOVA, analysis of variance; $M$, month.

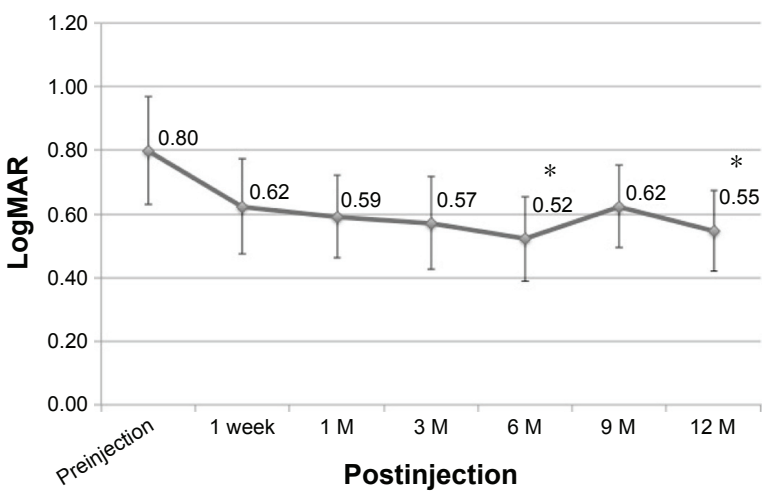

Figure 3 Changes in visual acuity in CRVO.

Note: $* P<0.05$, ANOVA versus preinjection.

Abbreviations: CRVO, central retinal vein occlusion; ANOVA, analysis of variance; $\log M A R, \log$ minimum angular resolution; $M$, month.

of IVR treatment have varied. Campochiaro et $\mathrm{al}^{10}$ reported that for CRVO patients, the IVR was administered six times during the introductory period, with an improvement of 13.9 letters in the Early Treatment Diabetic Retinopathy Study (ETDRS), and an IVR dosage of 8.8 times during 12 months. Brynskov et $\mathrm{al}^{13}$ administered IVR three times during the introductory period, with a median dosage of six times and an improvement of 2.6 letters in the ETDRS after 12 months. In the present study, there was a 0.25 improvement in the $\log$ MAR values with an average dosage of 3.4 times in a 12-month period for CRVO and an improvement of 12.5 letters in the ETDRS conversion units.

For BRVO, there was an improvement of 12.5 letters with an average of 8.1 injections during the period and six injections during the introductory period as reported by Tan et al. ${ }^{14}$ Brynskov et $\mathrm{l}^{13}$ reported three injections during the introductory period, with an improvement of 12.8 letters, and a median of five injections. Brown et $\mathrm{al}^{11}$ reported an improvement of 18.3 letters with an average of 8.4 injections,

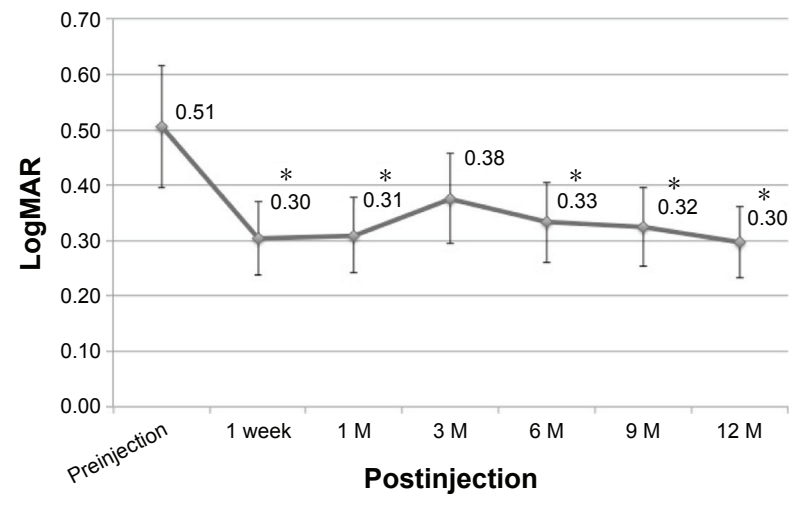

Figure 4 Changes in visual acuity in BRVO.

Note: $* P<0.05$, ANOVA versus preinjection.

Abbreviations: BRVO, branch retinal vein occlusion; ANOVA, analysis of variance; $\log M A R, \log$ minimum angular resolution; $M$, month. 
Table I Preinjection parameters that affected visual acuity after 12 months of the initial injection

\begin{tabular}{llll}
\hline $\begin{array}{l}\text { Preinjection } \\
\text { factor }\end{array}$ & $\begin{array}{l}\text { Crude } \\
\text { odds ratio }\end{array}$ & $95 \% \mathrm{Cl}$ & P-value \\
\hline Age & 0.991 & $0.914-1.074$ & 0.8198 \\
Sex & 1.8 & $0.318-10.203$ & 0.5066 \\
CMT & 2.003 & $0.997-1.009$ & $0.377 \mid$ \\
logMAR BCVA & 63.732 & $1.027-3,956.807$ & $0.0485 *$ \\
EZ continuity & 60.2144 & $0.131-409.738$ & 0.9796 \\
Ischemic & 0.381 & $0.065-2.222$ & 0.2834 \\
Preperiod & 0.972 & $0.759-1.246$ & $0.824 \mid$ \\
\hline
\end{tabular}

Note: *Good preinjection logMAR visual acuity was associated with a good visual acuity after 12 months of the initial injection $(P=0.0485$, logistic regression analyses).

Abbreviations: $\mathrm{Cl}$, confidence interval; $\mathrm{CMT}$, central macula thickness; logMAR $\mathrm{BCVA}$, logarithm of the minimum angle of resolution best-corrected visual acuity; EZ continuity, ellipsoid zone continuity.

with six injections during the introductory period. In the present study, we showed an improvement of 0.21 letters on the $\log$ MAR scale with 2.1 injections during a 12-month period for BRVO and an improvement of 10.5 letters in ETDRS conversion units.

Because the need for additional injections differed due to case backgrounds, we were unable to make simple comparisons, but it was possible that improvements in BCVA were similar to those previously reported for multiple injections during the introductory period, although we only used one injection during the same period in our study. Previous studies have recommended three injections during the introductory period, ${ }^{12,13,15}$ but if there are substantial improvements in BCVA, even with a low injection frequency during the introductory period, we propose that complications resulting from intravitreal injections as well as the burden of medical expenses resulting from multiple injections can be minimized, thus making the treatment more effective. Previous reports suggested that the standard for intravitreal reinjection is CMT $>300 \mu \mathrm{m}^{16-18}$ or $>250 \mu \mathrm{m},{ }^{14,19-22}$ but in our study, we set the standard for reinjection as a CMT of $300 \mu \mathrm{m}$ in order to further reduce the number of injections.

We identified preinjection parameters that resulted in a favorable BCVA after 12 months, which were predicted to be independent of preinjection BCVA. There was a good BCVA prognosis with a good preinjection BCVA. We believe that the good preinjection BCVA was a result of the limited extent of outer retina disorders. In the present study, we could not identify any relationship in the interval between the BCVA prognosis and the continuity of the ellipsoid zone prior to injection, but Hasegawa et $\mathrm{al}^{23}$ reported numerous cases where tears in the ellipsoid zone were recognized after cases where high reflective lines were observed from cysts existing after injection, with an additional relationship with the BCVA prognosis. By detailed observations following injection, the BCVA prognosis can, therefore, be predicted.

At all times 1 week after injection, the CMT significantly improved in both CRVO and BRVO compared with preinjection levels. Accordingly, we found that IVR structurally suppressed ME from its early stages regardless of the type of RVO. However, while there were improvements in BCVA for BRVO after 1 week of injection, the improvements were recognized in CRVO starting from 6 months after injection. While functions rapidly improved along with recovery from ME in BRVO, there was a time difference in improvement and ME recovery for CRVO.

We recognized recurrence of ME after 3 months of initial injections in many cases of CRVO, both within 3 months and at later times, with more cases of recurrence afterward with BRVO changes. Therefore, we were unable to suppress ME with a single injection for CRVO, and there were numerous cases requiring multiple injections. However, there were few cases of recurrence after the 3-month time point for CRVO. For cases of CRVO, a lack of recurrence of ME in the 3-month period following injection was indicative of a low possibility of later recurrence. Because there were few cases, additional cases should be studied to confirm the results of the current study.

In the present study, PRP was performed as soon as ischemia was diagnosed in cases with CRVO. In largescale study, there are reports of no reductions in injection frequency compared with simple ranibizumab therapy, even when IVR is combined with laser therapy. ${ }^{24}$ However, there is also a report that there was no decrease in the percentage of neovascular outbreaks in ischemic CRVO even when IVR was performed. ${ }^{25}$ Accordingly, there are many institutions in Japan treating CRVO that perform PRP as soon as ischemia is confirmed, using fluorescein angiography in order to prevent neovascular glaucoma. Although this examination is effective for IVR treatment of CRVO, we used this treatment, and there were no cases of neovascularization during the 1-year postinjection period.

IVR is widely used for age-related macular degeneration (AMD) and diabetic macular edema (DME), and three injections are recommended for AMD, while multiple injections are recommended during the introduction period for DME. ${ }^{26,27}$ We obtained positive results with a single injection and PRN for RVO, but there is still controversy whether a single injection is effective for AMD and DME. Unlike RVO, these are chronic diseases, and the control of edema and exudative changes is still insufficient after the first IVR; thus, multiple injections may be necessary for these diseases. ${ }^{26,27}$ 


\section{Conclusion}

In this study, we recognized a significant improvement in BCVA after 1 year using IVR with PRN and a single injection during the introductory period. However, because the study lasted for only 1 year, longer observational periods are necessary to confirm our conclusions. In addition, it may be possible to obtain even better efficacy and reduced injection frequency by using a combination of therapeutic methods.

\section{Disclosure}

The authors report no conflicts of interest in this work.

\section{References}

1. Mitchell P, Smith W, Chang A. Prevalence and associations of retinal vein occlusion in Australia. The Blue Mountains Eye Study. Arch Ophthalmol. 1996;114(10):1243-1247.

2. Klein R, Klein BE, Moss SE, Meuer SM. The epidemiology of retinal vein occlusion: the Beaver Dam Eye Study. Trans Am Ophthalmol Soc. 2000;98:133-141.

3. Rogers S, McIntosh RL, Cheung N, et al; International Eye Disease Consortium. The prevalence of retinal vein occlusion: pooled data from population studies from the United States, Europe, Asia, and Australia. Ophthalmology. 2010;117(2):313-319.

4. Campochiaro PA, Hafiz G, Shah SM, et al. Ranibizumab for macular edema due to retinal vein occlusions: implication of VEGF as a critical stimulator. Mol Ther. 2008;16(4):791-799.

5. Argon laser photocoagulation for macular edema in branch vein occlusion. The Branch Vein Occlusion Study Group. Am J Ophthalmol. 1984; 98(3):271-282.

6. Scott IU, Ip MS, VanVeldhuisen PC, et al; SCORE Study Research Group. A randomized trial comparing the efficacy and safety of intravitreal triamcinolone with standard care to treat vision loss associated with macular Edema secondary to branch retinal vein occlusion: the Standard Care vs Corticosteroid for Retinal Vein Occlusion (SCORE) study report 6. Arch Ophthalmol. 2009;127(9):1115-1128.

7. Ip MS, Scott IU, VanVeldhuisen PC, et al; SCORE Study Research Group. A randomized trial comparing the efficacy and safety of intravitreal triamcinolone with observation to treat vision loss associated with macular edema secondary to central retinal vein occlusion: the Standard Care vs Corticosteroid for Retinal Vein Occlusion (SCORE) study report 5. Arch Ophthalmol. 2009;127(9):1101-1114.

8. Noma H, Funatsu H, Mimura T, Shimada K. Macular sensitivity and morphology after intravitreal injection of triamcinolone acetonide for macular edema with branch retinal vein occlusion. Retina. 2012;32(9): 1844-1852.

9. Noma H, Mimura T. Macular sensitivity and morphology after intravitreal injection of triamcinolone acetonide for macular edema secondary to central retinal vein occlusion. Clin Ophthalmol. 2012;6:1901-1906.

10. Campochiaro PA, Brown DM, Awh CC, et al. Sustained benefits from ranibizumab for macular edema following central retinal vein occlusion: twelve-month outcomes of a phase III study. Ophthalmology. 2011; 118(10):2041-2049.

Clinical Ophthalmology

\section{Publish your work in this journal}

Clinical Ophthalmology is an international, peer-reviewed journal covering all subspecialties within ophthalmology. Key topics include: Optometry; Visual science; Pharmacology and drug therapy in eye diseases; Basic Sciences; Primary and Secondary eye care; Patient Safety and Quality of Care Improvements. This journal is indexed on Submit your manuscript here: http://www.dovepress.com/clinical-ophthalmology-journal
11. Brown DM, Campochiaro PA, Bhisitkul RB, et al. Sustained benefits from ranibizumab for macular edema following branch retinal vein occlusion: 12-month outcomes of a phase III study. Ophthalmology. 2011;118(8): 1594-1602.

12. Nghiem-Buffet S, Fajnkuchen F, Buffet M, et al. Intravitreal ranibizumab and/or dexamethasone implant for macular edema secondary to retinal vein occlusion. Ophthalmologica. 2014;232(4):216-222.

13. Brynskov T, Kemp H, Sørensen TL. Intravitreal ranibizumab for retinal vein occlusion through 1 year in clinical practice. Retina. 2014;34(8): 1637-1643.

14. Tan MH, McAllister IL, Gillies ME, et al. Randomized controlled trial of intravitreal ranibizumab versus standard grid laser for macular edema following branch retinal vein occlusion. Am J Ophthalmol. 2014;157(1): 237-247.

15. Berger AR, Cruess AF, Altomare F, et al. Optimal treatment of retinal vein occlusion: Canadian expert consensus. Ophthalmologica. 2015; 234(1):6-25.

16. Campochiaro PA, Wykoff CC, Singer M, et al. Monthly versus as-needed ranibizumab injections in patients with retinal vein occlusion: the SHORE Study. Ophthalmology. 2014;121(12):2432-2442.

17. Singer M, Tan CS, Bell D, Sadda SR. Area of peripheral retinal nonperfusion and treatment response in branch and central retinal vein occlusion. Retina. 2014;34(9):1736-1742.

18. Mylonas G, Sacu S, Dunavoelgyl R, et al; Macula Study Group. Response of retinal sensitivity to ranibizumab treatment of macular edema after acute branch retinal vein occlusion. Retina. 2013;33(6):1220-1226.

19. Campochiaro PA, Heier JS, Feiner L, et al; BRAVO Investigators. Ranibizumab for macular edema following branch retinal vein occlusion: six-month primary end point results of a phase III study. Ophthalmology. 2010;117(6):1102-1112.

20. Brown DM, Campochiaro PA, Singh RP, et al; CRUISE Investigators. Ranibizumab for macular edema following central retinal vein occlusion: six-month primary end point results of a phase III study. Ophthalmology. 2010;117(6):1124-1133.

21. Campochiaro PA, Sophie R, Pearlman J, et al; RETAIN Study Group. Longterm outcomes in patients with retinal vein occlusion treated with ranibizumab: the RETAIN Study. Ophthalmology. 2014;121(1):209-219.

22. Noma H, Miura T, Kuse M, Yasuda K, Shimura M. Photopic negative response in branch retinal vein occlusion with macular edema. Int Ophthalmol. 2015;35(1):19-26.

23. Hasegawa T, Masuda N, Ogata N. Highly reflective line in optical coherence tomography images of eyes with macular edema associated with branch retinal vein occlusion. Am J Ophthalmol. 2015;159(5):925-933.

24. Campochiaro PA, Hafiz G, Mir TA, et al. Scatter photocoagulation does not reduce macular edema or treatment burden in patients with retinal vein occlusion: the RELATE trial. Ophthalmology. 2015;122(7):1426-1437.

25. Brown DM, Wykoff CC, Wong TP, et al; RAVE Study Group. Ranibizumab in preproliferative (ischemic) central retinal vein occlusion: the rubeosis anti-VEGF (RAVE) trial. Retina. 2014;34(9):1728-1735.

26. Nguyen QD, Brown DM, Marcus DM, et al. Ranibizumab for diabetic macular edema: results from 2 phase III randomized trials: RISE and RIDE. Ophthalmology. 2012;119(4):789-801.

27. Lalwani GA, Rosenfeld PJ, Fung AE, et al. A variable-dosing regimen with intravitreal ranibizumab for neovascular age-related macular degeneration: year 2 of the PrONTO Study. Am J Ophthalmol. 2009; 148(1):43-58.

PubMed Central and CAS, and is the official journal of The Society of Clinical Ophthalmology (SCO). The manuscript management system is completely online and includes a very quick and fair peer-review system, which is all easy to use. Visit http://www.dovepress.com/ testimonials.php to read real quotes from published authors. 